prescription management; home visits; care coordination; and emotional support. Multiple perceived facilitators and barriers were identified, relating to: accessibility; continuity; GP's attitude and interpersonal skills; time and staffing levels; GP's involvement in diagnosis; and communication between primary and secondary care.

Discussion This study explored patients' views and experiences of the GP's role, and the perceived potential facilitators and barriers to its fulfilment. Further work is required to characterise a realistic model of continuous and accessible community palliative care.

\section{P-151 THE VALUE OF A NIGHT SERVICE FOR HOSPICE-AT- HOME: REASONS FOR MAKING CONTACT AT NIGHT}

Jo Clarke, Sue Varvel. Rennie Grove Hospice Care, Tring, UK

10.1136/bmjspcare-2018-hospiceabs. 176

Background People approaching the end of their lives have physical and psychological needs that should be met in a timely way, at any point during the night or day, and met in a way which addresses their needs and preferences. Crises at any time require prompt, safe and effective urgent care (NICE 2011).

Methods Rennie Grove Hospice Care (RGHC) provides 24/7 care, with a night service available from $9.15 \mathrm{pm}$ to $7.15 \mathrm{am}$. RGHC conducted an independent study to identify the value of the service, including reasons people make contact at night and how many calls could be settled without a visit. Over the study period (145 nights) each call was charted with demographic details, reason for call and outcome.

Results 550 calls were received, resulting in 335 visits. On seven nights there were no visits, the highest number in a night was nine, with an average 3.79 visits per night. Only those consenting to participate were analysed, reducing eligiblity to 351 calls and 208 visits. Of these, reasons for a night visit included symptom control (64\%), carer support (19\%), death (13\%), death verified by night team (9\%), message (7.5\%), planned visit (7\%) and 'other' (4\%). Major symptoms included pain (42\%), anxiety (32\%), nausea (17\%) and a small number with chest problems, bowel issues, pyrexia, syringe pumps. In 143 cases a visit was not necessary due to telephone reassurance (55\%), another service being more suitable $(12 \%)$, the team were too busy $(8 \%)$, and $12 \%$ were handed to the next shift.

Conclusions The night team plays a vital role in 24/7 care, with $40 \%$ of calls being managed without a visit. Visits were, in the main, for symptoms or problems concerning support. Death, for a Hospice at Home service also becomes a significant issue requiring a prompt and caring response.

\section{P-152 DOES A 24/7 HOSPICE AT HOME SERVICE PREVENT OR POSTPONE ACUTE HOSPITAL ADMISSIONS?}

Jo Clarke, Sue Varvel. Rennie Grove Hospice Care, Tring, UK

10.1136/bmjspcare-2018-hospiceabs.177

Background Most people wish to die at home but in England almost 50\% die in hospital, suggesting that more can be done to keep people at home. Some studies have shown this may be possible, especially with adequate support and good pain control.

Methods Rennie Grove Hospice Care (RGHC) carried out an independent study to identify the value of their night team in providing 24/7 care over a period of 145 nights. The study considered whether the provision of overnight care affected decisions to make hospital admissions. Data from a night nurse template recording all overnight visits, a review of 42 patient/carer records of those who had called the night team, a carer questionnaire $(n=87)$, carer interviews $(n=18)$ and staff interviews $(n=9)$, were analysed.

Results The night nurse template recorded five overnight admissions made during the study period, all for acute reasons and considered unavoidable. Review of 42 patient records showed 23 hospital/hospice admissions occurred, most during the daytime (18 to hospital, five to a hospice) of which only three were considered avoidable. The carer questionnaires showed 13 (16\%) of their cared-for persons had been admitted to hospital in the last few weeks of life, with most reporting the admission was necessary. Staff interviews identified a pride in supporting patients to stay at home, while information from carers gave a clear indication that RGHC nurses enabled their cared-for individual to remain at home; that contact with the night team helped prevent or postpone an admission; and without RGHC their alternative would have been $111 / 999$, district nurse or outof-hours GP.

Conclusions There are occasions when hospital admission is appropriate. However, there was clear evidence showing support from the night team could prevent or postpone hospital admissions.

\section{P-153 THE COST OF THE OVERNIGHT SERVICE AT RENNIE GROVE HOSPICE CARE VERSUS THE COST OF AN ACUTE ADMISSION}

Jo Clarke, Sue Varvel. Rennie Grove Hospice Care, Tring, UK

\subsection{6/bmjspcare-2018-hospiceabs. 178}

Background Rennie Grove Hospice Care (RGHC) runs a Hospice at Home service providing 24/7 care. An independent study calculated the cost of a RGHC visit and the total community cost of home care, including all health care professional (HCP), carer, and family member visits.

Methods Over a period of 145 days, 550 calls and 335 visits made to/by the night team were recorded, averaging 3.79 per night. The salary cost per hour for each nurse, plus organisational add-on costs, was calculated. To derive a total community cost, 35 families, considered by the nursing team to be able to consent, kept a diary for up to two-weeks, recording all HCP, carer and family support visits and duration of each visit. Seventeen diaries were returned. Descriptive analysis was used with the Statistical Package for the Social Sciences (SPSS v22). Costs were taken for HCPs and social care services from the Personal Social Services Research Unit data 2015/16

Results For 3.79 visits per night the cost per visit was $£ 195$ (taking into consideration that RGHC nurses travel in pairs). The 17 patient diaries covered a total of 177 days and 
showed a range of visits and complexity of care. RGHC staff provided $19 \%$ of the care at a cost of $£ 3295$; district nurses $13 \%$, cost $£ 2005$; and formal carers $55 \%$ at a cost of $£ 1344$. 23 GPs visits comprised $4 \%$ of all visits but $15 \%$ of total costs. Macmillan/Marie Curie nurses accounted for just 2\% of visits but $19 \%$ of cost as they stayed overnight. The entire cost of 177 days of care for 17 patients at end of life was $£ 11,814$; i.e. $£ 66.7$ per day as care was not needed every day of each diary period. The average length of the diaries was 10.4 days.

Conclusions The cost of home care seems acceptable, compared to the national average cost of a day in an inpatient specialist palliative care bed at $£ 397-£ 400$ (Data.Gov.UK 2015).

\section{P-154 EMERGENCY ADMISSIONS TO A HOSPICE: CAN HOSPICE AT HOME SERVICES REDUCE THESE?}

Kirsty Lewis, Aparajita Das. Countess Mountbatten Hospice, Southampton, UK

\subsection{6/bmjspcare-2018-hospiceabs.179}

Background Hospice at Home $(\mathrm{H} @ \mathrm{H})$ services aim to provide at-home care whilst dying, but these services are not universal and although studies have examined whether such services can prevent acute hospital admission, it is unclear whether they can prevent emergency hospice admissions (Buck, Webb, Moth et al., 2018; Ward, Graham \& Sixsmith, 2017).

Aim To investigate whether the introduction of a $\mathrm{H} @ \mathrm{H}$ service would reduce emergency hospice admissions, by analysing the needs of patients admitted as an emergency to a hospice inpatient unit.

Method All patients admitted as an emergency to this hospice, which does not currently offer a $\mathrm{H} @ \mathrm{H}$ service, are reviewed by the multi-disciplinary team at Mortality and Morbidity ( $\mathrm{M}$ and M) meetings. The data from this evaluation was collected between September 2016 and August 2017.

Results 190 emergency admissions took place during the above period. 93 patients (48.9\%) were admitted for complex symptom control, 52 patients $(27.4 \%)$ were admitted for end of life care, and 27 (14.2\%) were admitted for both complex symptom control and end of life care. Eleven patients $(5.8 \%)$ were admitted for multiple reasons including complex symptom control, end of life care, complex social situation and psychological distress. Seven patients (3.7\%) were admitted because of social crisis. At the $\mathrm{M}$ and $\mathrm{M}$ review it was agreed that a H@H service would not have prevented 122 admissions (64.2\%) but may have prevented 58 admissions (30.5\%). In 10 cases $(5.3 \%)$ it was unclear if $\mathrm{H} @ \mathrm{H}$ could have prevented admission.

Conclusion Most emergency admissions were for symptom control or multiple reasons, not end of life care alone; this may explain why only $30.5 \%$ of admissions could have been prevented with a $\mathrm{H} @ \mathrm{H}$ service. Patients choosing the hospice as their preferred place of death, or who were not imminently dying, will also affect the number of preventable admissions. This information on the needs of patients requiring emergency admission may help in the future development of a $\mathrm{H} @ \mathrm{H}$ service.

\section{P-155 INTRODUCING RAPID RESPONSE FOR COMMUNITY PALLIATIVE CARE, CLINICAL NURSE SPECIALISTS (CNS) TEAMS}

Geraldine Barry, Marika Killilea. North London Hospice, London, UK

10.1136/bmjspcare-2018-hospiceabs. 180

Background There is a national drive to support patients in their preferred place of choice and a need to reduce the number of unnecessary hospital admissions (Department of Health, 2008; National Palliative and End of Life Care Partnership, 2015). The community teams received over 1600 referrals last year. The triage team perform a telephone assessment, prioritising patients on need and transferring them to community clinical nurse specialists (CNSs). There was no formal pathway to respond to urgent needs of new and existing patients. It was unclear where to escalate urgent calls if all CNSs were occupied with planned visits. We decided to introduce a designated daily rapid response CNS into each of the teams to respond to the urgent referrals and reduce the number of patients who died before assessment.

Aims To introduce changes in working practices to respond to patients in a timely manner, with the intention to provide palliative care to more patients at home, thus supporting them in their preferred place of care (Calanzani, Higginson, Gomes, 2013).

Results The role of the rapid response CNS was defined and introduced into the three borough teams - each team identifies a rapid response CNS each day on the off duty. They are the first point of contact for the triage team and community administrators who frequently take calls from patients. The rapid response CNSs minimise planned visits for that day to ensure they have time to respond to urgent patient need.

Conclusion This was a challenge to traditional ways of working (Leary, Crouch, Lezard et al., 2008), however, involving the CNSs in developing the structure of the role and the pathway helped to develop a sense of ownership of the project. Although it is too early to formally evaluate there is a feeling of confidence and security for staff in the knowledge that their responsibilities are clearly defined. For the future we will examine responsiveness by introducing new activity codes to support data collection.

\section{P-156 URGENT RESPONSE: MEETING NEED, CHANGING CULTURE}

Nicola Griffiths, Fran Moffat, Sharon Hudson. Birmingham St Mary's Hospice, Birmingham, UK

10.1136/bmjspcare-2018-hospiceabs. 181

Background A Pilot Urgent Response Service was launched by the Hospice@ Home team due to it being identified that the nature of care needs for patients in the community were changing. More people required an urgent response for care crisis, symptom management, emotional distress and end of life care.

Aim The service aimed to support the Clinical Nurse Specialist and Primary Care Team in managing patients with urgent palliative care needs by coordinating a visit within two hours of a referral. The service was available to any patient already known to the Community Palliative Care Team (CNS, Hospice (a) Home and Day Hospice). 